\title{
Dos dimensiones de la estrategia "me too": consumidor y organización ${ }^{1}$

\author{
Two dimensions of the "me too" strategy: consumer and \\ organization
}

\author{
Ana María Arboleda Arango \\ Universidad Icesi, Colombia \\ amarboleda@icesi.edu.co
}

Artículo de reflexión recibido el 15/11/09 y aprobado el 31/05/10

\begin{abstract}
Resumen
Este documento analiza la estrategia " $m e$ too" desde dos dimensiones. Primero, como proceso cognitivo y, segundo, como una estrategia organizacional. La primera dimensión se trata desde la psicología a través de la categorización y generalización. La segunda se presenta desde ejemplos y razones estratégicas que llevan a las empresas a utilizar la imitación como herramienta competitiva. Finalmente, es claro que ambos procesos son complementarios como parte de la evolución de los mercados y el aprendizaje del individuo de las categorías de productos.

Palabras clave: Categorización, Generalización, Estrategia “me too”, Imitación.

Abstract

This document presents a two dimensional analysis of the "me too" strategy-- from a cognitive and strategic point of view. The first assumes a psychological perspective using concepts of categorization and generalization. The second presents examples and strategic reasons that led companies to use imitation as a competitive tool. Finally, it is clear that both processes are complementary and form part of the markets' growth and individuals' learning about product categories.
\end{abstract}

Key words: Categorization, Generalization, "Me too" strategy, Imitation.

1 Este artículo reune resultados de las investigaciones en psicología organizacional, realizadas por la autora durante los ańos 2008 y 2009, en la Universidad Icesi. 



\section{Introducción}

Mi hijo fue a la tienda a comprar un Bom bom bum; el Bom bom bum es rojo, el tradicional. Resulta que ahora además han salido otros dizque Plop, Plup... Entonces él (niño) le dice al señor (tendero) que le dé un Bom bom bum, y él (tendero) le da un Plup. Entones, mi hijo, que ni siquiera lee, le dijo, ese no es Bom bom bum. El (niño) identificó inmediatamente cual era... es muy difícil que uno utilice un producto continuamente y vaya a equivocarse (Arboleda, 2008: 42).

La estrategia "me too" se refiere a la construcción de una marca a partir de la imitación de las características de la marca líder en el mercado. Esta estrategia es utilizada por departamentos de mercadeo de compañías a través del mundo creando productos y marcas cuya imagen, uso y composición se guía por patrones de la categoría, que a su vez está determinada por el líder de la misma. Un producto o marca que utilizan esta estrategia se conocen como " me too". Es posible entender el uso de esta estrategia competitiva tanto desde la psicología como desde la administración estratégica. Así, este documento hace un análisis teniendo en cuenta, primero, estudios que explican la estrategia desde el comportamiento del consumidor y, segundo, la lógica organizacional para el uso de la estrategia.

Teniendo en cuenta la legislación colombiana este análisis no es trivial. A partir de la sentencia emitida por la Corte Suprema de Justicia colombiana en 2005 es posible entender que la imitación es una estrategia viable en Colombia siempre y cuando esta no lleve a la confusión del consumidor. La Corte es tajante en que la estrategia no debe causar confusión y en este sentido el uso y registro de marca es un aspecto clave en todos los sectores de la economía (2009). La lógica bajo la sentencia de imitación es que esta práctica incrementa la competencia entre las marcas y la poca diferenciación conlleva a diferencias de precio, luego, el principal beneficiado es el consumidor (Corte Suprema de Justicia y Sala de Casación Civil. Magistrado Ponente: Munar-Cadena, 2005). Adicionalmente, la reglamentación incentiva primero a que las marcas y características de las marcas sean registradas para así proteger la propiedad intelectual. Segundo, la imitación genera la necesidad de innovación, estrategia que sí lleva a la diferenciación entre las marcas.

La lógica de la estrategia “me too” es utilizar las características del líder para comunicar al consumidor que se están ofreciendo los mismos beneficios. Teniendo esto en cuenta, la viabilidad de la imitación, en términos de la jurisprudencia colombiana, genera permanentes debates. La controversia se da tanto por derechos de propiedad intelectual de acuerdo con los registros de marca (El Tiempo, 
2007), como por el cuestionamiento de las estrategias de comercialización de las marcas genéricas (Angell, 2004a, b). Pero, la presencia de productos que utilizan la estrategia "me too" puede tener consecuencias positivas o negativas. En el sentido positivo (para el consumidor), la estrategia permite reconocer rápidamente las características del producto que el consumidor espera. A nivel del mercado, incentiva la competitividad en la medida en que una mayor oferta de productos semejantes disminuye los precios y obliga al mejoramiento del producto base (Garattini, 1997).

Desde el punto de vista negativo, los diseńos de empaque desarrollados bajo la estrategia " $m e$ too" pueden generar confusión si no se tiene un adecuado manejo en recordación y visibilidad de la marca en el empaque (Alonso \& Arboleda, 2009). En términos del mercado, la disminución en los precios, podría disminuir la calidad de los productos si las organizaciones no contrarrestan la estrategia " $m e$ too" con innovación. Sin embargo y como se discutirá a través de este documento, las consecuencias positivas o negativas de la imitación encuentra su equilibrio entre el proceso cognitivo de categorización y la tendencia a la innovación en una categoría de productos. De esta manera, el análisis integral de la estrategia "me too" implica tener en cuenta dos dimensiones: el consumidor y el mercado.

Así, este documento analiza la estrategia " $m e$ too" primero como parte del proceso de toma de decisión del consumidor y, segundo, como una estrategia diseñada por las empresas de acuerdo con las características del mercado. El primer punto se elaborará compilando estudios que analizan la racionalidad de la imitación, desde el comportamiento del consumidor. Seguidamente, la dimensión del consumidor se complementa con una visión de mercadeo estratégico en la que se exponen las razones que llevan a las empresas a utilizar la imitación en sus productos.

\section{La estrategia "me too" desde el comportamiento del consumidor}

A nivel del consumidor el uso de la estrategia " $m e$ too" se basa en la capacidad cognitiva de categorización y generalización, procesos que se logran a partir de la familiaridad con las marcas y con la categoría de productos. Esta sección explica el proceso de categorización y generalización para luego entender cómo estos procesos cognitivos permiten y son la base para el uso de la estrategia " $m e$ too".

El proceso de categorización consiste en crear estereotipos, es decir, imágenes que se tienen en la mente acerca de algo específico y que contienen las caracterís-

1 Los procesos cognitivos en los que se construyen los conceptos de categorización y generalización son explicados por la psicología social como aquellos que permiten a un individuo reconocer su identidad a partir de la afiliación a un grupo y a partir de la diferenciación con otros grupos (Turner, 1975). Las teorías de cognición social transfieren los conceptos de la psicología social a la relación de un individuo con objetos (Levine \& Resnick, 1993). 
ticas básicas de lo que representa esa imagen. La creación e implementación de estereotipos es un proceso cognitivo automático (Fiske \& Taylor, 2008) que le permite al individuo entender, atribuir beneficios y defectos, y finalmente tomar decisiones de una manera más ágil. Otra expresión para esta imagen representativa de una categoría es un prototipo, algo que esboza las características de lo que representa un objeto de la categoría. Gracias al estereotipo, un producto no tiene la necesidad de reunir todas las características para ser categorizado. Por ejemplo, si el consumidor ve un empaque que es una botella, con la forma más estrecha en la parte superior, de unos $350 \mathrm{ml}$, de vidrio café-opaco, con tapa metálica, aunque no tenga marca probablemente al verlo va a pensar que es un embase de una cerveza.

Se puede llegar a la categorización a través de diferentes procesos dependiendo de la forma en que cada individuo aprende. El primero es analítico y consiste en juzgar los productos o marcas que no les son familiares al individuo partir de otros ya conocidos. El segundo es no analítico ya que de forma empírica el consumidor va aprendiendo de la categoría y experimentando la presencia de los atributos de los diferentes productos (Basu, 1993). Ambos procesos interactúan de tal forma que el individuo aprende las características de un producto que actúa como modelo guía. Los modelos permiten que los productos que más se parecen sean categorizados como de la misma familia (Cohen \& Basu, 1987). Al tener el concepto de "familia" o categoría (i.e., un grupo de productos con características semejantes) el individuo puede realizar una generalización.

La generalización consiste en atribuir las características aprendidas de un objeto (i.e., producto o marca) a los demás de la categoría. Es decir, de acuerdo con su experiencia, el individuo entiende que ciertas características son las que representan la categoría. Luego al generalizar, esas características, beneficios y defectos, las atribuirá a los demás elementos que correspondan a la categoría. Por lo tanto, el consumidor aprende de los productos de acuerdo a lo que ya tiene en su mente y es por eso que no se instruye sobre cada producto individualmente sino que lo categoriza para lograr un mejor entendimiento. De lo contario, en el caso de no tener un conocimiento previo, el individuo deberá construirlo (e.g., para qué sirve el producto, qué beneficios y defectos tiene) lo que requerirá un mayor esfuerzo cognitivo (Sujan, 1985). Esto es consistente con la idea de que en etapas tempranas del aprendizaje del producto los individuos hacen un mayor esfuerzo cognitivo y se ven más interesados por la búsqueda de información (Van Waterschoota, Sinha, Van Kenhove, \& De Wulfd, 2008).

Un conocimiento previo, o experticia, con respecto a la categoría y sus marcas implica que el individuo tiene ya más información de las mismas (Liberman 
\& Montgomery, 1998) y puede más inferencias acerca de aspectos específicos del producto (Sujan, 1985; Sujan \& Dekleva, 1987) como el uso, desempeño o imagen. Así, en la medida que el consumidor aprende las características básicas de las categorías puede acceder (cognitivamente) a otros productos generalizando las características ya aprendidas. Por lo tanto son los productos líderes de la categoría, probablemente aquellos que han estado en el mercado por más tiempo, los que generan parámetros para reconocer las características del producto. Entonces al ver productos similares, el consumidor generaliza este aprendizaje y da atributos semejantes a productos semejantes en su apariencia aunque los reconozca como marcas diferentes (Miaoulis \& D'Amato, 1978; Warlop \& Alba, 2004).

Los procesos cognitivos de categorización y generalización ocurren porque las personas sienten la necesidad de evitar la ambigüedad, de recibir información que les permita tomar decisiones de manera rápida y clara (Vermeir, Van Kenhove, \& Hendrickx, 2002). Lo que la teoría Gestalt llamaría necesidad de "cierre". Es decir, los proceso cognitivos permiten al individuo comprender, analizar y tomar decisiones teniendo en cuenta el todo y no elementos aislados (Donis, 2000). Por ejemplo, en el verbatim con el que comienza este documento, el niño ha tenido una experiencia previa en la que ha asociado el empaque de cierto color, textura, tamańo, y marca con un sabor y una experiencia. ${ }^{2}$ Gracias a la asociación de estos elementos él sabe que ese producto corresponde a la categoría de bombones y además reconoce la marca particular (probablemente entienda que son semejantes). La elaboración de un todo (una Gestalt) es natural cuando el consumidor conoce la categoría y sus características. En caso contrario la persona debe buscar información acerca del producto para poder tomar una decisión.

La necesidad de obtener información además está mediada por el tipo de categoría para la cual el consumidor esté tomando la decisión de compra. Categorías que implican un mayor nivel de involucramiento, como por ejemplo los medicamentos, deben proveer información clara y legible en el empaque (Foxman, Muehling, \& Berger, 1990; Vermeir, et al., 2002). Además, el tiempo dedicado a la compra depende de la categoría de producto. Productos de categorías "importantes", como medicamentos, tienen para los consumidores un proceso de elección más cuidadoso. Esto es consecuencia de las implicaciones que puede tener comprar o consumir el producto equivocado. El acto de compra resulta ser más importante si la percepción de riesgo incrementa, y así mismo incrementa el nivel de involucramiento (Bloch \& Richins, 1983; Foxman, et al., 1990).

2 De acuerdo con la Gestalt, la percepción de los objetos se da de manera integral, sin dar mayor importancia a unas cualidades que a otras; hay una percepción integral del todo, en: Metzger, W. (1928), "Certain Implications in the Concept of 'Gestalt". The American Journal of Psychology, 40(1): 162 - 166. 


\section{El “me too" es una generalización}

La estrategia " $m e$ too" se basa en la de asignación de atributivos a los productos (categorización) y la extrapolación de estos atributos a productos semejantes (generalización). Esto implica que la categoría de productos es relativamente familiar para el consumidor. Es decir, un producto desarrollado bajo la estrategia "me too" corresponde a categorías de productos donde el consumidor busca tomar decisiones relativamente ágiles. Al buscar un "me too" los individuos buscan reconocer elementos claves en el producto habitual y eso les permite ahorrar tiempo en el momento de compra (Ruiz de Maya, 1997; Schmitt \& Simonson, 1997). Esto implica que el consumidor desde un comienzo conoce lo que va a comprar, es decir, realiza una compra repetida. Durante las compras repetidas la persona tiene en mente las características del objeto que busca y por lo tanto entiende y responde con mayor facilidad a símbolos (Schiffman \& Kanuk, 2005).

Dada la comprensión de símbolos, la principal simbología utilizada en el diseño de los empaques de productos "me too" son el color y la forma. La consistencia que tiene una categoría en el uso de los colores es útil para el consumidor porque es lo que le permite reconocer las características del producto. Los colores tienen valores simbólicos generados tanto por factores fisiológicos e.g., efecto de la frecuencia de honda (Gorn, Chattopadhyay, Yi, \& Dahl, 1997) como por el aprendizaje a partir de la experiencia con la categoría (Arboleda, 2008). Los colores son estímulos que tienen un significado y por lo tanto una consecuencia emocional (Leichtling, 2003). Así mismo, la forma es relevante porque los individuos tienen consciencia acerca del significado de las formas. De acuerdo con su experiencia de aprendizaje los individuos tienen la capacidad de entender conceptualmente el uso y las características del objeto a partir de su forma (Campbell, 1995). La forma es otro elemento (además del color) que facilita la categorización y por lo tanto el proceso cognitivo de transferir información (Ludwing, 1995). Por este motivo, el significado de un objeto que tiene una forma particular tiende a atribuirse a otro con una forma semejante.

Adicionalmente, la comprensión de los símbolos, es decir el significado de las características visibles del producto, está estrechamente relacionada con la importancia que tiene el mensaje o el producto para la persona (Bloch $\&$ Richins, 1983). En caso de un bajo involucramiento (poca relevancia) la información se hace trivial y no hay un compromiso por parte del consumidor con esta (VargasBianchi, 2003). En casos de un menor involucramiento o menor familiaridad con la categoría de productos hay una mayor posibilidad de confusión (Foxman et al., 1990) por que el individuo no conoce el significado de las características del producto. Al no tener un prototipo, es decir, no tener claras las características 
que debe exhibir el producto, el consumidor no puede saber qué lo diferencia de otros con características semejantes o diferentes. El no reconocimiento de las características del empaque, ya sea porque no han sido aprendidas o porque son demasiadas cosas nuevas para aprender del diseño y del producto, son factores que generan confusión (Mitchell \& Papavassiliou, 1999; Mitchell \& Walsh, 2005). De esta manera, la confusión es un tema crítico al hablar de productos " $m e$ too".

\section{La confusión del consumidor por el uso de la estrategia " $m e$ too"}

Cuando existe familiaridad con la categoría los consumidores tienen la capacidad de diferenciar productos que son similares por su empaque e imagen, pero con marcas diferentes. Es decir, a través de la generalización los consumidores atribuyen características percibidas de la marca conocida a las otras marcas, siendo claro para los consumidores que las marcas comparten atributos pero son diferentes (Balabanis \& Craven, 1997; Miaoulis \& D’Amato, 1978). Por lo tanto, si la percepción general del consumidor acerca de productos con apariencias similares es diferente para cada producto, no se puede acusar de confusión (Schmitt \& Simonson, 1997).

La confusión del consumidor se ha definido como un fallo cognitivo (Alonso \& Arboleda, 2009; Turnbull, Leek, \& Ying, 2000) y sólo en pocos casos se ha explicado la confusión teniendo en cuenta el proceso emocional del consumidor (Jacoby, Speller, \& Berning, 1974; Kohli \& Thakor, 1997). En síntesis, la confusión es un error en el proceso de toma de decisión que ocurre por falencias en el conocimiento de la categoría o en la capacidad para asimilar la información. La confusión puede tener consecuencias sobre lo emocional, generando emociones negativas como la frustración y el enojo (Mitchell \& Walsh, 2005).

Así mismo, la confusión puede ocurrir por tres razones. Primero, porque no se conocen las características de la categoría y el consumidor hace una inferencia errónea (Balabanis \& Craven, 1997). Segundo, porque no se presta atención a la marca, siendo esta un elemento esencial en la reducción de la probabilidad de confusión (Alonso \& Arboleda, 2009). Tercero, porque la marca o la categoría realizan cambios importantes y constantes en su diseño generando exceso de información, haciendo difícil el aprendizaje del significado de las características (Mitchell \& Papavassiliou, 1999; Mitchell \& Walsh, 2005). ${ }^{3}$

El primer punto se refiere al proceso de categorización y generalización, siendo esta la razón que sustenta el uso de la estrategia "me too" como una práctica que puede evitar la confusión. Por ejemplo, si el consumidor se encuentra con un embase plástico, alargado, de textura suave, con una silueta estilizada, que

3 En este documento no se discutirán las consecuencias emocionales de la confusión. 
se desatapa levantando un extremo desde una pestańa (no rosca) y con colores pastel $^{4}$ quizás va a saber que se trata de un producto de aseo personal. La comprensión de los atributos a través de los símbolos son claves para el aprendizaje de la categoría (Kapferer, 1995; Turnbull, et al., 2000), e incluso facilitan la memorabilidad de la marca desde la infancia (Macklin, 1996).

Es decir, la experiencia de aprendizaje permite hacer una clara categorización, bajo la cual tiene sentido que los productos que hacen parte de esta "familia" sean relativamente similares (Arboleda, 2008). Si no lo fuesen el consumidor no reconocería una marca como parte de esa categoría, podría incluso atribuirlo a otra categoría (a la que sí sea semejante) y hacer un uso inadecuado. Por ejemplo, tener productos de aseo del hogar, como limpiadores de pisos, con embases semejantes a los de aseo personal podría llevar a una compra y uso equivocado. Este ha sido un factor crítico en los medicamentos en donde se ha observado que las enfermeras o farmaceutas pueden cometer errores si no se tienen colores consistentes para una misma categoría (Kenagy \& Stein, 2001).

El segundo elemento que puede generar confusión es el manejo o la lectura inadecuada de la marca. Los consumidores habituales de una categoría o de una marca tienen una menor posibilidad de confundirse ante una estrategia " $m e$ too" mientras que en el caso de los consumidores esporádicos la decisión depende del nivel de importancia y el riesgo atribuido a la compra. En la medida en que estos factores aumentan así mismo aumenta la búsqueda de información (Foxman, et al., 1990) y disminuye la confusión.

Existe particular atención a la información que se brinda en el caso de los medicamentos. Por ejemplo, la FDA (Food and Drug Administration) ha impuesto normas para los medicamentos de venta libre indicando que deben proveer en sus empaques la información necesaria para evitar la confusión. Esta información consiste en los componentes y sus concentraciones, contraindicaciones, precauciones e indicaciones de consumo. Toda esta información debe ser visible, legible, clara y escrita en un vocabulario simple (Kenagy \& Stein, 2001). Estas normas pretenden evitan que la estrategia " $m e$ too" genere confusión en esa categoría de productos (Spencer, 2002).

Así, cuando hay una menor familiaridad con la categoría, la comunicación a través de texto resulta ser un poco más eficaz que la gráfica (Grisaffe $\&$ Shellabarger, 1997) y especialmente el uso adecuado de la marca resulta ser prioritario para evitar la probabilidad de confusión (Alonso \& Arboleda, 2009; Cohen, 1991). Incluso, los consumidores no tienen buena memoria acerca de

4 Ver: Russel Russel, D. (1990), Libro de los colores pasteles; del azul; del amarillo; del rojo; y del verde, 1 ed. Barcelona: Editorial Gustavo Hill, S.A. Para una mayor explicación en el significado de los colores pastel. 
características sutiles del diseño y los colores secundarios del empaque (Green, 2004), lo que recuerdan con facilidad son las características básicas del empaque como la marca. Por lo tanto, si los nombres de las marcas "me too" no suenan ni se ven parecidos y la etiqueta no está visualmente cargada de información, la confusión se puede evitar en gran proporción (Diamond, 1981; Kearney \& Mitchell, 2001; Kohli \& Thakor, 1997).

En general, el nombre del producto ayuda a evitar la confusión y es un argumento válido para establecer parámetros de diferenciación evidentes para el consumidor. Cuando el nombre del producto es claro, visible, bien marcado (sobresaliente) y diferente al de productos similares (en este caso productos " $m e$ too"), la probabilidad de confusión es mínima porque el consumidor sabe qué está llevando (Schmitt \& Simonson, 1997). Así las organizaciones responsables deben garantizar la legibilidad de la marca "me too" (Kenagy \& Stein, 2001). Sin embargo, para determinar la claridad con la que se presenta la marca y conocer la probabilidad de confusión en el consumidor es recomendable realizar experimentos en los que se comprueba el uso correcto de símbolos y colores para identificar una marca como diferente de otras que hacen parte de una misma categoría (Alonso \& Arboleda, 2009; Cohen, 1991; Cohen \& Basu, 1987).

El tercer punto que puede generar confusión es el exceso de información (Mitchell \& Papavassiliou, 1999; Mitchell \& Walsh, 2005; Sproles, 1985). La proliferación de marcas o el cambio constante en los diseńos de los empaques genera una mayor densidad de información que debe ser absorbida por el individuo. Por lo tanto, teniendo en cuenta la limitación natural del individuo (i.e., bounded-rationality) en cuanto a la capacidad de asimilación de información en un mismo momento (Simon, 1979), el exceso de información puede ser un factor de confusión. Este exceso de información puede ocurrir como consecuencia de la proliferación de marcas, sobrecarga de información en los empaques o cambio frecuente en los diseños de los empaques. Estos factores hacen más compleja la categoría (Huffman \& Kahn, 1998) y por lo tanto más difícil para el consumidor interpretar la simbología de los colores y formas del empaque.

Algunos autores (Loken, Ross, \& Hinkle, 1986; Miaoulis \& D’Amato, 1978) argumentan que la estrecha semejanza en las características físicas entre productos es un factor de confusión (i.e., el uso de la estrategia " $m e$ too"). Sin embargo, si se tienen en cuenta las razones de confusión antes mencionadas, es precisamente la consistencia y la estabilidad en las características lo que evita la confusión. La psicología ha logrado una mejor comprensión de los procesos cognitivos siendo claro que la generalización de las características es una consecuencia de la capacidad para formar estereotipos y categorías, algo que por el contrario 
facilita la toma de decisión. Lo importante, entonces, es la clara diferenciación de marcas dentro de una misma categoría. Adicionalmente, es por esto que la estrategia "me too" ocurre en categorías donde las características del producto típico ya están establecidas.

En términos generales, al hablar de una categoría madura, la confusión del consumidor se pude controlar en la medida en que se siguen códigos similares en el interior de la categoría teniendo una clara diferenciación de marcas. Siendo así, no es necesariamente el uso de la estrategia "me too" la que causa confusión en el consumidor (sin decir que no lo hace). La confusión puede ser causa de las estrategias de mercadeo en general. Cuando la estrategia de mercadeo no busca la construcción de la marca sino que por el contrario la marca se diluye en estrategias orientadas al precio, el consumidor busca menos información del producto, se distrae de la marca, y toma la decisión con base en el precio, sin tener una clara diferenciación de los productos disponibles (Clancy \& Trout, 2002). La presencia de productos similares acompañado de precios bajos puede motivar la compra en consumidores impulsivos (Balabanis, et al., 1997). Es decir, en estos casos los precios bajos pueden generar preferencia evitando estudiar otras diferencias. Cuando los pecios y las características son iguales, el producto "me too" no es interpretado como uno que persuade al consumidor de comprar una marca inferior. Sin embargo, si hay un mayor costo la similitud puede ser percibida negativamente (Warlop, et al., 2004).

En síntesis, la estrategia "me too" se basa en el proceso cognitivo a través del cual el consumidor aprende a través de la experiencia las características de un producto estableciendo un prototipo, es decir define las características de la categoría. Al generalizar las características se espera que productos con funciones semejantes muestren diseńos semejantes. La comprensión de estas semejanzas es un factor positivo debido a que permite al consumidor comprender extender atributos y beneficios básicos a productos de la misma categoría. En esta dinámica la marca juega un papel importante seńalando que aunque los atributos pueden ser semejantes la marca no es la misma y será el rol de la marca señalar otros atributos diferenciadores. Sin embargo, pensando que los productos de una categoría han logrado un desarrollo relativamente estándar en cuanto a su apariencia y funcionalidad, para el consumidor será claro que la oferta no cambia mucho de una marca a otra. Es por esto, que la estrategia "me too" es propia de mercados maduros en donde el producto está relativamente desarrollado y existe una demanda por el producto establecida. El uso de la estrategia " $m e$ too" en mercados realmente innovadores en donde las características no son estables sí puede ser un factor negativo y dañino para el consumidor porque no tendrá 
tiempo para tomar decisiones utilizando el aprendizaje de la categoría. Teniendo esto en cuenta, a continuación se analizará el uso de la estrategia " $m e$ too" desde el punto de vista gerencial.

\section{Fundamentos gerenciales para el uso de la estrategia " $m e$ too"}

El uso la estrategia "me too" corresponde a categorías maduras en las que las características del producto están ya desarrolladas y las estrategias se centran en obtener mayor participación de mercado. El " $m e$ too" se desarrolla bajo la idea de que lo que el líder de la categoría ha hecho ha funcionado en términos de posicionamiento y penetración en el mercado. El líder ha logrado la construcción en la mente de los consumidores de unas características y funcionalidades particulares a un producto. De acuerdo con esto, el consumidor puede suponer que un producto semejante debe verse y funcionar de forma semejante (Streeter, 2006).

Teniendo en cuenta que el líder previamente ha familiarizado a los consumidores con las características del producto, los productos "me too" se caracterizan por requerir poca inversión publicitaria (Carpenter \& Nakamoto, 1990). En la etapa introductoria, la marca pionera, que normalmente es la líder de la categoría, se encarga de que el conocimiento de ese nuevo producto o servicio llegue a los consumidores potenciales (Liberman \& Montgomery, 1998). Pasada esta etapa de altos gastos en investigación $\&$ desarrollo y en mercadeo, se llega a la etapa de crecimiento, que es cuando surgen los competidores. Así, los competidores son aquellos productos que siguen la pauta establecida por el líder, adaptándose a lo que ya los consumidores han aprendido y esperan del producto. Los seguidores son los que incursionan en un mercado después de que ya existe la información del producto y el tipo de consumidor (Berkowitz, Hartley, Kevin, $\&$ Rudelius, 2003).

La otra característica de la estrategia "me too" es que reduce los precios ya sea directa o indirectamente. Directamente el " $m e$ too" puede entrar a competir a un menor precio. Indirectamente, el " $m e$ too" aumenta la oferta lo cual genera una reducción en los precios del mercado. Las marcas competidoras crean imitaciones para captar una porción del mercado que ya es consumidora, es decir, la compañía que establece la estrategia " $m e$ too" supone que el producto tiene una demanda clara, relativamente estable. Los competidores además suponen que la demanda puede ser ampliada al aumentar la oferta (Angell, 2004a; Grant, 2008).

Manteniendo otros aspectos constantes, el aumento en la oferta reduce los precios del producto en el mercado. Sin embargo, habitualmente el líder de la categoría continúa teniendo la mayor participación de mercado porque se le atribuyen mayores niveles de calidad. Es decir, dada la relativa semejanza entre 
marcas de una categoría, el argumento de calidad debe ser el factor diferenciador para controlar la caída de los precios (Lee, 2004). Por lo tanto, es importante analizar la importancia que tiene para el consumidor de ese producto particular, cómo es la sensibilidad al precio vs la calidad esperada. En muchos casos el consumidor no está dispuesto a reducir características como la exclusividad, la calidad, la variedad de funciones segundarias y el diseńo a cambio de una reducción del precio (Johnson, 1989). En esos casos una estrategia "me too" no sería recomendable.

La dinámica de aumento en la oferta y reducción en los precios para un posterior aumento en la demanda, hace parte del ciclo de vida de los productos, siendo propia de mercados maduros en donde la tecnología del producto y su fabricación ya ha sido desarrollada. El siguiente paso en el desarrollo del mercado de un producto es la expansión en la distribución y esta se logra en la medida en que incrementa la demanda por el producto (Grant, 2008). Es decir, en la medida en que hay un mayor número de consumidores interesados en acceder al producto. En este momento, cuando los costos de innovación y desarrollo son bajos, el producto "me too" absorbe las características ya desarrolladas para establecer una oferta semejante. La capacidad de absorción se refiere a la habilidad que tiene la empresa para identificar y adquirir conocimientos y usarlos para introducir productos con propósitos comerciales (Cohen \& Levinthal, 1990).

En la medida en que las compañías se preparan para incursionar en mercados internacionales observan los parámetros establecidos por los participantes (o específicamente el líder) de esa categoría (Root, 1994). El nuevo competidor tiene la posibilidad de recopilar información existente en el mercado y aplicarla bajo la figura del " $m e$ too". Lanzar al mercado un producto o servicio similar proporciona cierta seguridad debido a que ya se conocen las características esperadas por el consumidor, así la probabilidad de fracaso disminuye. Teniendo esto en cuenta, el uso de la estrategia "me too" es gerencialmente válida no solo porque "facilita" el acceder rápidamente a mercados internacionales, sino también porque permite ampliar la oferta de marcas a nivel nacional. Consistentemente, las empresas utilizan la estrategia " $m$ e too" porque los requerimientos de tiempo e inversión para la creación de un producto es menor que realizar nuevos desarrollos (Menrad, 2001). Por lo tanto, es una estrategia viable tanto para las grandes multinacionales como para pequeñas y medianas empresas que ofrecen productos ya conocidos para un segmento y necesidades específicas.

En general, las empresas que practican la estrategia "me too" carecen del know-how y/o de recursos tecnológicos y financieros para una labor intensiva en investigación y desarrollo. En algunos casos tampoco tienen la capacidad 
para gastar grandes sumas de dinero en información específica o actividades de marketing como si lo puede hacer una empresa pionera (Menrad, 2001). Así pues, los desarrollos tecnológicos que no están patentados, o que cumplen con el periodo de protección de la patente, son absorbidos por los competidores. Para este momento el consumidor tendrá un conocimiento de la tecnología y características del producto, lo que facilita la introducción de nuevas marcas con características de diseño, concepto y contenido similares. En algunos casos, la imitación puede limitarse a las características de contenido y propiedades del producto, que por lo tanto conlleva a productos con una funcionalidad similar. Pero la principal característica de la estrategia es la similitud visual, que le permita al consumidor tomar una decisión ágil con base en la apariencia. Esto se conoce bajo el concepto de tradedress. El tradedress es el "vestido", el conjunto de elementos que hacen parte de la apariencia general de un producto o de las características de los establecimientos en los que se ofrece un servicio; el tradedress permite que un producto o servicio sea visualmente diferente del resto (Espitia, 2006; Warlop \& Alba, 2004).

\section{La estrategia " me too" se expande a través de las industrias}

La estrategia "me too" no es propia de una categoría de productos o de un solo sector. Por el contrario, se han encontrado evidencia de que la estrategia " $m e$ too" se extiende a través de muchas categorías de la economía. A continuación se citan unos ejemplos.

La estrategia se utiliza con frecuencia en servicios tales como bancos, supermercados de cadena, televisión por cable, computadores. En estos casos la estrategia "me too" resulta ser útil porque permite unificar el tipo de tecnología y así garantizar un mayor desarrollo beneficiando a los usuarios del servicio/ producto (Fernández, 1994). Por ejemplo, el hecho de que todos los fabricantes de televisores produzcan sus equipos utilizando la tecnología LED $^{5}$ permite que esta se desarrolle más rápido. A los ojos del consumidor las nuevas pantallas de las diferentes marcas serán relativamente semejantes y será la preferencia del consumidor por características secundarias al producto o por el valor dado a la marca lo que le permita finalmente tomar una decisión de compra.

La estrategia " $m e$ too" también está presente en la industria automotriz, donde los diseños de las casas automotrices competidoras son rápidamente imitados. Tal es el caso de Mitsubishi que creó la Mitsubishi Raider, su propia versión de la Dodge Dakota (Autoweek, 2004). El uso de las características que el líder

5 Tecnología que permite la conducción de energía a través de diodos. La sigla se toma por su definición en inglés: light-emiting diode (LED). 
impone son rápidamente utilizadas por los competidores; esto también ocurre en el mundo de la moda en prendas de vestir. En el momento en que el trabajo de un diseñador se convierte en moda ${ }^{6}$ es porque diferentes marcas y fabricantes utilizan en sus prendas características similares (Schmitt \& Simonson, 1997).

Otro sector donde se presenta la estrategia " $m e$ too" a nivel mundial es el de alimentos "funcionales" (Menrad, 2001). Los alimentos funcionales son diseñados buscando beneficios positivos sobre la salud y tienen la capacidad de reducir el riesgo de ciertas enfermedades crónicas. Los productos "me too" se utilizan en este caso en categorías como repostería, pasta y galletas y para cada una de estas se usan colores y símbolos que comunican al consumidor que está consumiendo un producto saludable. En esta categoría es posible ver el uso consistente de verde, imágenes de granos, y leyendas asociadas a la salud. Por otro lado, los alimentos tradicionales y de consumo masivo como por ejemplo el aceite de cocina, la pasta, la gaseosa, los jugos también guardan características relativamente semejantes en sus empaques en cuanto a color y forma al interior de cada una de estas categorías (Arboleda, 2008).

En la industria farmacéutica los medicamentos "me too" son el principal negocio (Angell, 2004b). En este sector se ha utilizado por mucho tiempo esta estrategia; tanto que en la literatura el uso del concepto "me too" muchas veces se asocia al uso de genéricos. ${ }^{7}$ Los productos genéricos son aquellos que salen al mercado después de que el laboratorio innovador lanza el desarrollo de una nueva molécula. Los productos genéricos pueden ser de dos tipos, aquellos que se ofrecen como una marca con una composición semejante a la marca líder y aquellos que ofrecen la misma composición que el líder pero dando relevancia al laboratorio fabricante.

Los medicamentos que son una copia de la fórmula original, que hacen el "desarrollo" de un genérico, también se conoce como Copycats. Por ejemplo Claritin (marca original) y Clarinex (copia), ambos de Schering-Plough (Spencer, 2002). Por otro lado, aunque la literatura relaciona la estrategia "me too" principalmente con el uso de genéricos, conceptualmente la estrategia "me too" va más allá del uso de componentes semejantes; implica seguir patrones propios

6 Si nos referimos a la definición matemática, la moda es donde encontramos la mayor frecuencia, la mayor ocurrencia de eventos. Quizás un buen ejemplo del uso de la estrategia " $m e$ too" es observar un curso de universitarios. Sus prendas de vestir, accesorios, cortes de cabello serán similares siempre que pertenezcan a un grupo (categoría) comunicando consistentemente sus preferencias.

7 En el caso de los productos genéricos, es importante recalcar que el uso de nombres genéricos para el producto no se puede proteger porque por lo general son nombres de componentes o drogas específicas. Si los nombres genéricos se protegieran habría más confusión porque sólo una marca tendría el derecho de utilizarla, haciendo que los otros productos no pudieran seńalar que clase de medicamento y que componentes tiene Schmitt, B., \& Simonson, A. 1997. Marketing y estética ( $1^{\text {a }}$ ed.). Ediciones Deusto, Madrid. 
de la categoría (principalmente del líder) y por lo tanto se puede entender a través del uso de una apariencia similar (tradedress).

Así, en muchos casos la estrategia "me too" no es la copia de la composición o la tecnología de otro producto directamente, sino de ciertas características claves. Por esta razón, al medir las percepciones de las personas acerca del el grado de similitud de varios productos y servicios ofrecidos en 46 categorías se encontraron que en 40 de las categorías se tienen marcas que los consumidores asocian como parecidas. Entre éstas están las tarjetas de crédito, tales como: Visa y Mastercard; algunos productos para el cuidado del pelo, como L'Oréal y Clairol; librerías, como Barnes \& Nobles y Borders; algunas aerolíneas como United y American, cremas de dientes, Colgate y Crest entre otras categorías (Trout \& Clancy, 2002). Estos resultados se dieron en gran medida por el uso relativamente estandarizado de factores claves (atributos y beneficios) que muestran las diferentes marcas de una categoría a los consumidores.

Otros ejemplos del uso de la estrategia "me too" a nivel mundial son Home Depot y Lowe`s (Streeter, 2006); Clorox-Purex (Carpenter \& Nakamoto, 1990); Tic-Tac - Dynamints-Mighty Mints (Miaoulis \& D'Amato, 1978); Shire US - Barr Laboratories, Inc., (medicamentos) (Shirk, Durkin, \& BassIII, 2003); Silver Toe - Gold Toe (Calzoncillos); Starter - Converse (Ropa e indumentaria deportiva); Opium - Omni (perfumes; Tyco - Lego (Juguetería); Schwinn - Ross (Instrumentos deportivos, Bicicleta estática); Playboy - Playmen (Revistas); Tylenol PM - Excedrin PM (Medicamentos) (Schmitt \& Simonson, 1997); Whirlpool - General Electric (Electrodomésticos); AT\&T - MCI (Servicios de Telecomunicaciones); Toyota - Honda (Automóviles); Hitachi - Sony (Electrodomésticos) (Trout \& Clancy, 2002); Crestor de AstraZeneca y Mevaco de Merck (medicamentos que reducen el nivel de colesterol) (Angell, 2004b); Pepsi y Coca-Cola (Carpenter \& Nakamoto, 1990); Danone-Alpina (El Tiempo, 2007).

Teniendo en cuenta los ejemplos anteriores, es claro que la estrategia " $m e$ too" es ampliamente utilizada a través de los sectores de la economía. Esta consistencia en el uso de la estrategia "me too" permite entender mejor que ella hace parte del desarrollo del mercado en el cual debe haber un constante balance entre la innovación y la imitación. La estrategia " $m e$ too" es pertinente en un mercado donde las características del producto ya estén desarrolladas y sea necesario mejorar la oferta para que ese desarrollo pueda llegar a más segmentos de la población. Es decir, debe ser un mercado relativamente maduro con cierto potencial de crecimiento en términos de demanda.

Así, si se analiza desde un continuo en el tiempo, la estrategia "me too", es decir, el momento de imitación, permite consolidar el desarrollo de los productos 
y construir la demanda para los desarrollos tecnológicos ya logrados. Al encontrar la saturación del mercado, la necesidad de satisfacer nuevas necesidades, la convergencia de nuevas tecnologías que faciliten otros desarrollos, será momento para la innovación. Por esta razón, es importante mirar la estrategia "me too" dentro del proceso evolutivo de competencia organizacional. Es decir, en el continuo de la dinámica competitiva (Iwai, 1984) en donde hay un momento para imitar y otro para innovar, el primero permite la consolidación de lo existente, el segundo lleva al mejoramiento incremental o al cambio radical de la oferta.

Un proceso organizacional competitivo debe tener ambos momentos (Aghion, Harris, Howitt, \& Vickers, 2001; Bessen, 2006; Bowie, 2005; Eiji, Tetsushi, \& Keijiro, 2005; Girard, 1990). La dinámica entre la imitación y la innovación, significa que existe un momento para reforzando las características ya adquiridas y un momento para adquirir nuevas capacidades competitivas. La limitación a uno de los dos obstruiría la dinámica competitiva de la organización (Segerstrom, 1991; Verona, 1999; Wendy \& Safarian, 2007).

\section{Conclusiones y futuras investigaciones}

En la comprensión e implementación de la estrategia "me too" se deben tener en cuenta dos perspectivas: el proceso cognitivo del consumidor y la decisión organización en términos de competitividad. Este documento resume ambos puntos planteando que la estrategia " $m e$ too" es la adecuada en la medida que el consumidor tiene un conocimiento claro de la categoría y la organización entra a competir en un mercado maduro en el que las características ya están establecidas.

En términos del consumidor, la confusión es un efecto relevante que se discute en torno al uso de la estrategia " $m e$ too". Lo que proporciona la estrategia "me too" es estabilidad en las características de la categoría, siendo esto un hecho que hasta cierto punto puede evitar la confusión si consideramos que el constante cambio de empaques o diseños en los productos confunde al consumidor. Por otro lado, la confusión que sí pueda generar la similitud entre empaques debe ser controlada por los fabricantes a través del manejo adecuado de la marca. Es claro que es responsabilidad del fabricante el utilizar correctamente los colores, formas y diseños propios de la categoría, así como señalar al consumidor que es una marca diferente.

En términos del mercado, el uso de la estrategia " $m e$ too" puede tener un efecto negativo sobre las marcas como consecuencia de una guerra de precios. La guerra de precios es una dinámica destructiva para las marcas ya que se utiliza el precio como estrategia diferenciadora en defecto de otros argumentos. Futuras investigaciones podrían encontrar estas dos perspectivas (la estrategia "me too" vs 
estrategia de precio) para determinar en qué medida su relación en el largo plazo puede generar una destrucción en la industria y no un incentivo a la innovación (como escape a la imitación).

En el mercado hay una dinámica permanente entre la imitación y la innovación (Eiji et al., 2005), dinámica que se acelera en la medida en que la industria y los mercados se hacen más competitivos. Por otro lado, al pensar en el consumidor la imitación proporciona una estabilidad cognitiva, un tiempo lo suficientemente largo como para aprender bien las características del producto, pero la innovación permite satisfacer necesidades diferentes. Incluso las dificultades de la innovación radican en que el nuevo producto puede no ajustarse a al aprendizaje que ha logrado el individuo de la categoría y por lo tanto puede no comprender o no necesitar dicha innovación. Es decir, existe una congruencia entre ambos niveles, el mercado y el consumidor.

La consistencia entre ambos niveles es un proceso que no ha sido explorado. Normalmente, la administración estratégica se encarga de comprender la dinámica a nivel del mercado y la psicología (a través del comportamiento del consumidor) analiza la capacidad de asimilación y satisfacción de necesidades del individuo. Las decisiones organizacionales relacionadas con el uso de la estrategia "me too" o la creación de productos realmente nuevos debe tener en cuenta que la madurez del mercado y tamańo de la demanda está relacionada con la capacidad cognitiva que el individuo tiene para aprender de y usar los nuevos productos.

Claramente, existe una brecha entre la administración estratégica y la psicología cognitiva que implica un trabajo conjunto con una metodología de estudio multinivel (Dansereau \& Yammarino, 2005). El integrar estas dos disciplinas permitiría entonces comprender la pertinencia de la estrategia " $m e$ too" o por el contrario la oportunidad del lanzamiento de productos nuevos. Por otro lado, esta integración puede facilitar a los empresarios una toma decisión integral, teniendo en cuenta que el desarrollo del mercado (imitación o lanzamiento de nuevos productos) está relacionado con la capacidad que tiene el individuo de absorber (cognitivamente) nuevos productos.

\section{Bibliografía}

Aghion, P., Harris, C., Howitt, P., \& Vickers, J. (2001). Competition, Imitation and Growth with Step-by-Step Innovation. The Review of Economic Studies, 68(3), 467-492. 
Alonso, J. C., \& Arboleda, A. M. (2009). Responsible designs: avoiding confusion in categories with "me too" products. Trabajo presentado en the Latin American Research Consortium (LARC).

Angell, M. (2004a). Excess in the pharmaceutical industry. Canadian Medical Association or its licensors, 171(12), 1451-1453.

Angell, M. (2004b). The truth about the drug companies : how they deceive us and what to do about it (1st ed.). New York, Random House.

Arboleda, A. M. (2008). Percepciones del color y de la forma de los empaques: una experiencia de aprendizaje. Estudios Gerenciales, 24(106), 31-45.

Autoweek (2004). “Mitsu's Me Too: '06 Raider”. Business Source Premier, 54, 1-6.

Balabanis, G., \& Craven, S. (1997). "Consumer confusion from own brand look-alikes: an exploratory investigation”. Journal of Marketing Management, 13, 299-313.

Basu, K. (1993). "Consumers' Categorization Processes: An Examination with Two Alternative Methodological Paradigms". Journal of Consumer Psychology, 2(2), 97-121.

Berkowitz, E. N., Hartley, S. W., Kevin, R. A., \& Rudelius, W. (2003). Marketing.

Bessen, J. M., Eric (2006). "Sequential Innovation, Patents, and Imitation". Unpublished Working Paper. Institute for Advanced Study, School of Social Science, Economics

Bloch, P. H., \& Richins, M. L. (1983). "A theoretical model for the study of product importance perceptions". Journal of Marketing, 47, 69-81.

Bowie, J. I. (2005). Innovation, imitation, legitimacy and deviance in the design of graphical trademarks in the United States, 1884-2003. Unpublished Ph.D., The University of Arizona, United States, Arizona.

Campbell, J. (1995). Shape Properties, Experience of Shape and Shape Concepts. Philosophical Issues, 7, 351-363. 
Carpenter, G. S., \& Nakamoto, K. (1990). "Competitive Strategies for Late entry into a Market with a Dominant Brand”, Management ScienceVol, 36, 1268-1278.

Clancy, K., \& Trout, J. (2002). “Brand confusion”. Harvard Business Review, 3.

Cohen, D. (1991). “Trademark Strategy Revisited". Journal of Marketing, 55(3), 46-59.

Cohen, J. B., \& Basu, K. (1987). "Alternative Models of Categorization: Toward a Contingent Processing Framework”. The Journal of Consumer Research, 13 (4), 455-472.

Cohen, W., \& Levinthal, D. (1990). "Absorptive capacity: A new perspective on learning and innovation". Administrative Science Quarterly, 35, 128-152.

Corte Suprema de Justicia y Sala de Casación Civil. Magistrado Ponente: Munar Cadena, P. O. (2005). Ref. Expediente 4018. Bogotá, Distrito Capital.

Dansereau, F., \& Yammarino, F. J. (2005). "Multi-level issues in strategy and methods". En Elseiver (Ed.), Research in multi-level issues (Vol. 4, pp. 197-237): Elseiver.

Diamond, S. A. (1981). Trademark Problems and How to Avoid Them, Revised Edition, . Chicago: Crain Communications.

Donis, D. A. (2000). "Elementos básicos de la comunicación visual”. In D. A. Donis (Ed.), La sintaxis de la imagen (14 ed.). Buenos Aires: Gustavo Gili. Eiji, Y., Tetsushi, S., \& Keijiro, O. (2005). "Time path in innovation, imitation, and growth: the case of the motorcycle industry in postwar Japan". Journal of Evolutionary Economics, 15(2), 169.

El Tiempo, C. e. (2007, Miércoles 12 de septiembre). "Danone inicia batalla jurídica contra Alpina por varios productos”. Portafolio,

Espitia, M. F. (2006). "Trade dress, una grieta abierta en la protección de derechos de propiedad industrial” Recuperado Mayo 24, de 2006, http://www.marcasur. com/es/opinion imprimir.asp?id=466 
Fernández, D. (1994). "Understanding Intellectual Property Rights Retrieved" Mayo 20, de 2006, http://www.ifla.org/documents/infopol/copyright/ferd1.htm

Fiske, S., \& Taylor, S. (2008). Social Cognition, from Brains to Culture McGrawHill Humanities.

Foxman, E. R., Berger, P. W., \& Cole, J. A. (1992). "Consumer Brand Confusion: A Conceptual Framework”. Psychology and Marketing, 9(March/April), 123-141.

Foxman, E. R., Muehling, D. D., \& Berger, P. W. (1990). "An Investigation of factors contributing to consumer brand confusion". Journal of Consumer Affairs, 24(1. Summer), 170-184.

Garattini, S. (1997). "Are Me Too drugs justified?” Journal of Nephrology, 10 (6), 283-294.

Girard, R. (1990). "Innovation and Repetition". SubStance, 19(2/3), 7-20.

Gorn, G. J., Chattopadhyay, A., Yi, T., \& Dahl, D. W. (1997). "Effects of color as an executional cue in advertising: They're on the shade". Management Science, 43(10), 1387-1400.

Grant, R. M. (2008). Contemporary Strategy Analysis (Sixth edition ed.): Blackwell Publishing Ltd.

Green, M. (2004). "Visual expert human factors: Color in trademark and tradedress disputes". 1- 6. Recuperado de www.visualexpert.com

Grisaffe, D. B., \& Shellabarger, S. (1997). "Consumer comprehension of efficacy data in four experimental over-the-counter label conditions". Drug Information Journal, 31, $937-961$.

Huffman, C., \& Kahn, B. E. (1998). "Variety for Sale: Mass Customization or Mass Confusion?” Journal of Retailing, 74(4), 491-513.

Iwai, K. (1984). "Schumpeterian dynamics: An evolutionary model of innovation and imitation". Journal of Economic Behavior \& Organization, 5(2), 159-190. 
Jacoby, J., Speller, D. E., \& Berning, C. A. (1974). "Brand Choice Behavior as a Function of Information Load: Replication and Extension". Journal of Consumer Research (pre-1986), 1(February), 33-42.

Johnson, M. D. (1989). "The Differential Processing of Product Category and Noncomparable Choice Alternatives". The Journal of Consumer Research, 16(3), 300-309.

Kapferer, J.-N. (1995). "Stealing brand equity: measuring perceptual confusion between national brands and 'copycat' own-label products". Marketing And Research Today May, 96-102.

Kearney, I., \& Mitchell, V.-W. (2001). "Measuring consumer brand confusion to comply with legal guidelines". International Journal of Market Research, 43(1), $85-91$.

Kenagy, J. W., \& Stein, G. C. (2001). "Naming, labeling and packaging of pharmaceuticals". American Journal of Health-System Pharmacy, 58(21), 2033-2041.

Kohli, C., \& Thakor, M. (1997). "Branding consumer goods: insight from theory and practice”. Journal of Consumer Marketing, 14, 206-219.

Lee, T. H. (2004). "Me Too' Products - Friend or Foe?". The New England Journal of Medicine, 350(3), 211-212.

Leichtling, C. (2003). "How color affect marketing". The TABS Journal, 26(3), 23-31.

Levine, J. M., \& Resnick, L. B. (1993). "Social foundations of cognition”. Annual Review of Psychology, 44(1), 585.

Liberman, M., \& Montgomery, D. B. (1998). "First-movers (dis)advantage: retrospective and link with the resource-based view". Strategic Management Journal, 19(12), 1111-1125.

Loken, B., Ross, I., \& Hinkle, R. L. (1986). "Consumer Confusion of Origin and Brand Similarity Perceptions". Journal of Public Policy and Marketing, 5, 195-211. 
Ludwing, K. (1995). “Shape properties and perception”. Philosophical issues, 7 , 326-350.

Macklin, M. C. (1996). "Preschoolers' Learning of Brand Names from Visual Cues”. Journal of Consumer Research (pre-1986), 23(3), 251-261.

Menrad, K. (2001). "Market and marketing of functional food in Europe". Journal of Food Engineering, 56, 181-188.

Metzger, W. (1928). "Certain Implications in the Concept of Gestalt". The American Journal of Psychology, 40(1), 162-166.

Miaoulis, G., \& D’Amato, N. (1978). "Consumer Confusion \& Trademark Infringement”. The Journal of Marketing, 42(2), 48-55.

Mitchell, V.-W., \& Papavassiliou, V. (1999). "Marketing causes and implications of consumer confusion". The Journal of Product and Brand Management, 8(4), 319.

Mitchell, V.-W., \& Walsh, G. (2005). "Towards a Conceptual Framework of E-Confusion". American Marketing Association. Conference Proceedings, 16, 44.

Ruiz de Maya, S. (1997). "El comportamiento del consumidor en marketing: Del método científico a su posición en la empresa”. Cuadernos de Administración 2-24.

Russel, D. (1990). Libro de los colores pasteles; del azul; del amarillo; del rojo; $y$ del verde (1 ed.). Editorial Gustavo Hill, Barcelona.

Schiffman, L. G., \& Kanuk, L. L. (2005). Comportamiento del consumidor (8 ed.). Pearson, México.

Schmitt, B., \& Simonson, A. (1997). Marketing y estética (1a ed.). Ediciones Deusto, Madrid.

Segerstrom, P. S. (1991). "Innovation, Imitation, and Economic Growth". The Journal of Political Economy, 99(4), 807-827.

Shirk, J., Durkin, T.-G., \& BassIII, K. (2003). "Pharmaceutical look-alikes". ClientTimes, 1-4. 
Simon, H. A. (1979). "Rational Decision Making in Business Organizations. The American Economic Review, 69(4), 493.

Spencer, H. M. (2002). "The RX-to-OTC switch of Claritin, Allegra, and Zyrtec: An unprecedented FDA response to petitioners and the protection of public health". American University Law Review, 51, 999-1050.

Sproles, G. B. (1985). "From Perfectionism to Fadism: Measuring Consumers' Decision-Making Styles". Proceedings, American Council on Consumer Interests, 79-85.

Streeter, W. W. (2006). “The Me-Too trap. [Editor's column]”. ABA Banking Journal, XCVII (1), 4.

Sujan, M. (1985). "Consumer Knowledge: Effects on Evaluation Strategies Mediating Consumer Judgments". The Journal of Consumer Research, 12(1), 31-46.

Sujan, M., \& Dekleva, C. (1987). "Product Categorization and Inference Making: Some Implications for Comparative Advertising". The Journal of Consumer Research, 14(3), 372-378.

Trout, J., \& Clancy, K. J. (2002). "Brand confusion”. Harvard Business School Publishing Corporation. .

Turnbull, P. W., Leek, S., \& Ying, G. (2000)." Customer Confusion: The Mobile Phone Market". Journal of Marketing Management, 16(January-April), 143-163.

Turner, J. C. (1975). "Social comparison and social identity: Some prospects for intergroup behaviour”. European Journal of Social Psychology, 5(1), 1-34.

Van Waterschoota, W., Sinha, P. K., Van Kenhove, P., \& De Wulfd, K. 2008. "Consumer learning and its impact on store format selection". Journal of Retailing and Consumer Services, 15: 194-210.

Vargas-Bianchi, L. (2003). "Procesamiento de información y familiaridad de marca”. Análisis 30, 225 - 240 
Vermeir, I., Van Kenhove, P., \& Hendrickx, H. (2002). "The influence of need for closure on consumer's choice behavior”. Journal of Economic Psychology, 703-727.

Verona, G. (1999). "A Resource-Based View of Product Development". The Academy of Management Review, 24(1), 132-142.

Warlop, L., \& Alba, J. W. (2004). "Sincere Flattery: Trade-Dress Imitation and Consumer Choice”. Journal of Consumer Psychology, 14 (1\&2), 21-27.

Wendy, D., \& Safarian, A. E. (2007). Making the transition from imitation to innovation: An enquiry into the technological capabilities of China's firms: International Tax Program, Institute for International Business, Joseph L. Rotman School of Management, University of Toronto. Toronto. 
